\title{
The first known Mira-type variable star in IC 1613
}

\author{
R. Kurtev ${ }^{1}$, L. Georgiev ${ }^{2}$, J. Borissova ${ }^{3}$, W. D. Li $^{4}$, A. V. Filippenko ${ }^{4}$, and R. R. Treffers ${ }^{4}$ \\ 1 Department of Astronomy, Sofia University, and Isaac Newton Institute of Chile, Bulgarian Branch, \\ 1164 Sofia, Bulgaria \\ 2 Instituto de Astronomía, Universidad Nacional Autónoma de México, Apartado postal 70-264, 04510 México, \\ D.F., México \\ e-mail: georgiev@astroscu.unam.mx \\ 3 Institute of Astronomy, Bulgarian Academy of Sciences, and Isaac Newton Institute of Chile, Bulgarian Branch, \\ 72 Tsarigradsko Chaussèe, 1784 Sofia, Bulgaria \\ e-mail: jura@haemimont.bg \\ 4 Department of Astronomy, University of California, Berkeley, CA 94720-3411, USA \\ e-mail: (wli, alex, rtreffers) @astro.berkeley.edu
}

Received 2 May 2001 / Accepted 14 August 2001

\begin{abstract}
King et al. (1999) discovered Nova 1999 in IC 1613 at the Lick Observatory. Both Fugazza et al. (2000) and Borissova et al. (2000) questioned this classification, because they were able to detect the star on images obtained in previous years. In infrared frames taken on Oct. 15, 1998, Nova 1999 has $(J-K)=1.14$ and $K=14.69$ mag. Our light curve study, based primarily on 92 unfiltered Lick images, suggested that the object could be a Mira-type variable with a period of 640.7 days. This period is very close to that obtained by Fugazza et al. (2000) - 631 days. The star is overluminous with respect to the period-luminosity (PL) relation derived by Feast et al. (1989) for Mira variables in the LMC. At longer periods ( $P>400$ days), many LMC Miras show such behavior and the PL relation appears to break down. It is possible that the situation in IC 1613 is similar. An optical spectrum obtained with the Keck-II telescope shows features typical of M3Ie or M3IIIe stars. We conclude that the star is a normal long-period M-type Mira variable, the first such star confirmed in IC 1613.
\end{abstract}

Key words. galaxies: individual: IC 1613 - galaxies: Local Group - galaxies: stellar content - stars: variables: general

\section{Introduction}

IC 1613 is a faint irregular member of the Local Group. The galaxy was discovered by Wolf (1906) with the 16-inch Bruce refractor at Heidelberg. Baade (1928), using plates taken with the 16-inch Bergedorf reflector, classified it as a Magellanic-Cloud-type galaxy.

The variable stars in IC 1613 have been investigated by Sandage (1971), Carlson \& Sandage (1990), and Freedman (1988a). Eight Wolf-Rayet stars are suspected (Armandroff \& Massey 1985) and one of them (WO3) is surrounded by nebulae. The light curves of Cepheids and other variable stars in Field A of IC 1613, obtained with CCD unfiltered photometry, have been analyzed by Antonello et al. (1999). Later Antonello et al. (2000) and Mantegazza et al. (2001) observed another three fields in the galaxy (Fields B, C, and D) to detect short-period Cepheids and to obtain good light curves for Fourier decomposition. In all four fields (A-D) they found a total of more than 300 variable stars of different types. Very recently, Dolphin et al. (2001) presented Hubble Space

Send offprint requests to: R. Kurtev, e-mail: kurtev@phys.uni-sofia.bg
Telescope WFPC2 VI photometry of a field in the halo of IC 1613 and found 13 RR Lyrae stars and 11 Cepheids. Using different distance indicators they found the best distance modulus of IC 1613 to be $\mu_{0}=24.31 \pm 0.06$.

King et al. (1999) discovered Nova 1999 in IC 1613 with the 0.8-m Katzman Automatic Imaging Telescope (KAIT; Li et al. 2000; Filippenko et al. 2001) at the Lick Observatory. The new object is located at $\alpha(2000)=$ $1^{\mathrm{h}} 04^{\mathrm{m}} 48^{\mathrm{s}} .97, \delta(2000)=+2^{\circ} 05^{\prime} 28^{\prime \prime} .8$. In the infrared (IR) frames taken on 1998 October 15 at the National Mexican Astronomical Observatory "San Pedro Martir", Nova 1999 has $(J-K)=1.14$ and $K=14.69 \mathrm{mag}$ (Borissova et al. $2000)$. Its presence in the images taken one year before the discovery and its low observed brightness during outburst call into question its classification as a nova. Fugazza et al. (2000) also noted that the nova falls in their Field B (Antonello et al. 2000), and its coordinates are coincident within the uncertainties with those of their variable V2950B. This star was observed four years before the announcement of the detection as a nova. Fugazza et al. (2000) found a period of 631 days for optical variations. These authors also noted a $B V R I$ measurement of this star obtained by Freedman (1988b), dating back to 1984 . 
Table 1. Uncertainty of KAIT photometry.

\begin{tabular}{rc}
\hline$R$ & $\sigma(R)$ \\
\hline 18.0 & 0.12 \\
18.5 & 0.13 \\
19.0 & 0.17 \\
19.5 & 0.23 \\
20.0 & 0.33 \\
20.5 & 0.52 \\
\hline
\end{tabular}

\section{Observations and data reduction}

\subsection{Photometry}

Unfiltered photometry of Nova 1999 was obtained during the interval 1998 July 25 - 2000 January 4 ( 90 CCD frames) with KAIT, as a byproduct of monitoring IC 1613 for supernovae. The images were obtained with an Apogee $512 \times 512$ pixel AP7 CCD camera. The scale at the Cassegrain focus of KAIT is $0.8^{\prime \prime}$ pixel $^{-1}$ and the total field of view is $6 ! 8 \times 6 ! 8$.

Additional CCD $B V R$ frames were taken on 1999 September 16, November 3, December 8, 2000 August 4, and 2001 July 15 with the 2-m Ritchey-Chretién (RC) telescope of the Bulgarian National Astronomical Observatory Rozhen with a Photometrics $1024 \times 1024$ pixel CCD camera. The scale at the Cassegrain focus is $0.33^{\prime \prime}$ pixel $^{-1}$ and the total field of view is $5 ! 6 \times 5 ! 6$. Stellar photometry of the frames taken with the 2-m telescope was performed with the point-spread function fitting routine ALLSTAR available in DAOPHOT II (Stetson 1993). Reduction of instrumental magnitudes to the standard $B V R$ system was accomplished using a few dozen observations of Landolt (1992) standard stars on each night.

Instrumental stellar magnitudes from the unfiltered KAIT observations were also derived with ALLSTAR available in DAOPHOT II (Stetson 1993). Using standard $R$ magnitudes and $V-R$ colors of the variable star and twelve local standard stars in the field obtained from Rozhen $B V R$ photometry, KAIT unfiltered instrumental magnitudes were transformed into Cousins $R$. A detailed discussion of the procedure can be found in Riess et al. (1999). The precision of the KAIT observations as a function of magnitude is given in Table 1.

The IR data were acquired with the infrared camera "CAMILA" with a NICMOS3 $256 \times 256$ pixel detector attached to the 2.1-m telescope of the National Mexican Observatory. The scale is 0. . 85 pixel $^{-1}$, resulting in a field size of about $3.6 \times 3.6$. A set of $J K$ frames was taken on 1998 October $13-15$. The seeing during these observations was $1^{\prime \prime}-1$.' 2 , with stable photometric conditions. Stellar photometry of the frames was done using DAOPHOT II (Stetson 1993). Twelve UKIRT standard stars (Cassaly \& Hawarden 1992) were measured before and after the nova observations.

\subsection{Spectroscopy}

Spectroscopic observations were made with the Low Resolution Imaging Spectrometer (LRIS; Oke et al. 1995) on the Keck-II 10-m telescope. Two spectra of the program star were obtained in photometric conditions on 1999 December 15 . The seeing varied from $1^{\prime \prime}$ to $1^{\prime \prime} 4$, and the slit width was $1^{\prime \prime}$. A 300 grooves $\mathrm{mm}^{-1}$ grating blazed at $5000 \AA$ gave a wavelength range of 3900-8900 $\AA$ $\left(2.5 \AA\right.$ pixel $\left.^{-1}\right)$, with minimal second-order contamination beyond $7600 \AA$. The spectral resolution was $9.9 \AA$ ( FWHM of the $[\mathrm{O} \mathrm{I}] \lambda 6300$ night-sky line). The exposure time for each spectrum was $300 \mathrm{~s}$. Both spectra were obtained at the parallactic angle of $130^{\circ}$ (Filippenko 1982) at an airmass of 1.11 .

The spectra were flux-calibrated using the standard star $\mathrm{BD}+17^{\circ} 4708$ (Oke \& Gunn 1983) at an airmass of 1.06. The flux standard was also used to remove telluric absorption lines through appropriate division. The two exposures were combined equally after scaling the first by a factor of 1.07. Cosmic rays and/or sky-line glitches were removed in the regions $6552.5-6660 \AA$, 4097.2-4100 $\AA$, and $4470 \AA$ (a single pixel). From our final merged spectrum, we derive $V R I$ magnitudes of the variable star.

\section{Photometric behavior}

Figure 1 shows the $R$-band light curve of Nova 1999, using the observations of Freedman (1988b), Fugazza et al. (2000), the Rozhen 2-m RC telescope, the transformed KAIT data, and the $R$ magnitude derived from the Keck spectrum. Initially the star was suspected to be a nova in IC 1613 by King et al. (1999). Its long-term photometric behavior, however, is typical of Mira-type variable stars.

We searched for the best period using a least-squares periodogram analysis by means of the phase dispersion minimization (PDM) task available in IRAF, and with a period-finding program based on the Lafler \& Kinman (1965) "theta" statistic. The result is 640.7 days. This value is near the upper limit for Miras. The mean light curve from all observations is displayed in Fig. 2. The amplitude of the star is more than $2.5 \mathrm{mag}$ in $R$, also typical for this type of red variable.

The infrared photometry gives $(J-K)=1.14$ and $K=14.69 \mathrm{mag}$ on JD 2451100 . As can be easily seen from Fig. 1, at this moment the $K$ magnitude of the star is somewhat less but close to its average $K$ magnitude. Taking into account that the amplitudes of Miratype variables are minimal in the infrared (not more than $1 \mathrm{mag}$ ), it is interesting to check whether this object lies on the Mira period-luminosity $(\mathrm{PL})$ relation found by Feast et al. (1989) in the LMC: $M_{K}=-3.47 \log (P /$ day $)+$ 0.78. According to this equation, the expected $M_{K}$ is -8.96 mag. For comparison, if we use the average observed $K$ magnitude and an adopted distance modulus of $24.31 \pm 0.06 \mathrm{mag}$ (Dolphin et al. 2001), we obtain $M_{K}=-9.62 \mathrm{mag}$. Thus, the star appears to be overluminous by about 0.7 mag with respect to the PL relation 


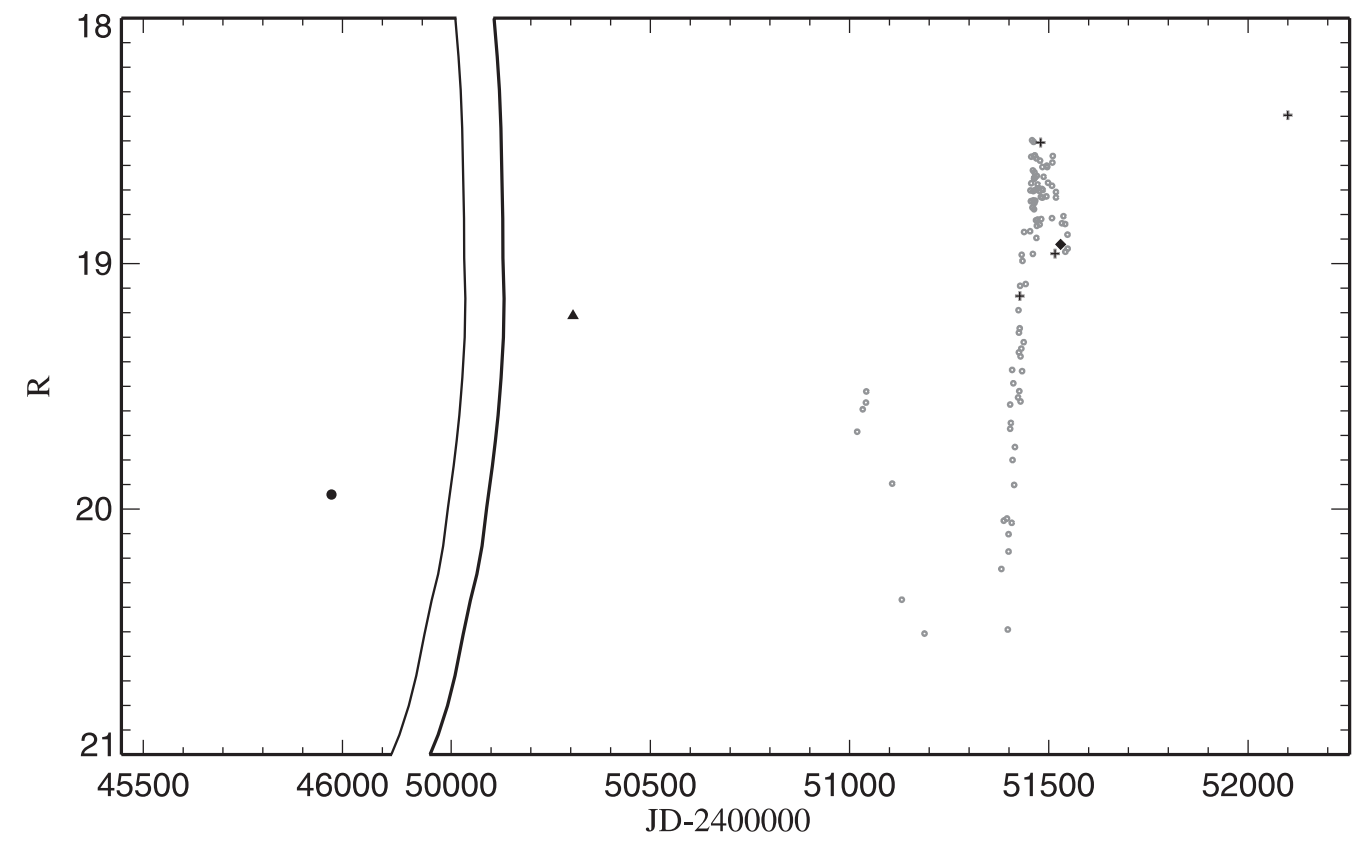

Fig. 1. The long-term photometric behavior of the Mira-type variable in IC 1613 ("Nova 1999"). Gray circles are KAIT observations transformed to $R$, crosses represent Rozhen observations, and the filled diamond is the $R$ magnitude derived from the Keck-II spectrum. The Freedman (1988b) magnitude of 19.94 on JD 2445973.88 (filled dot) and the Fugazza et al. (2000) magnitude of 19.21 on JD 2450305.90 (filled triangle) are also shown.

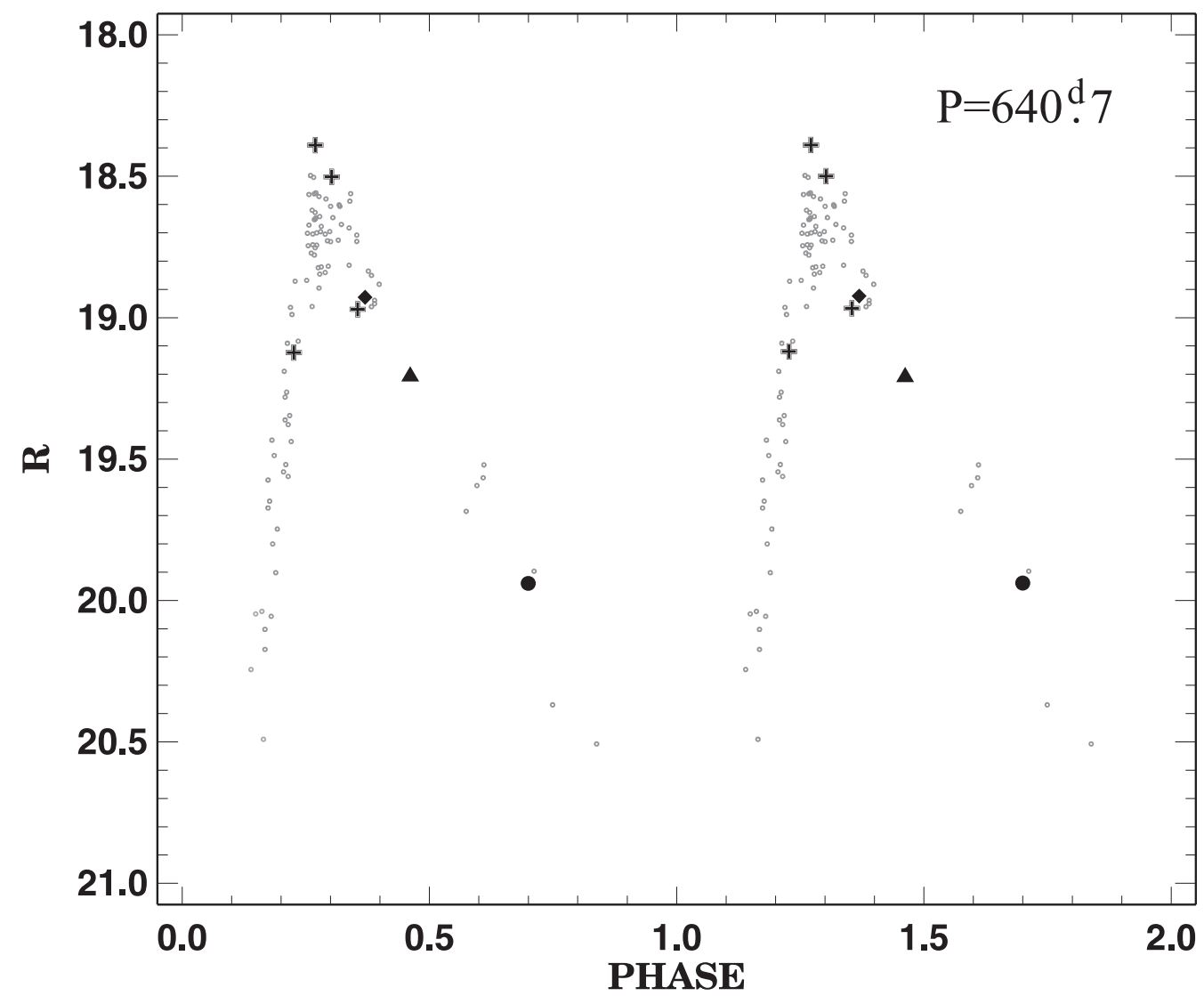

Fig. 2. The mean $R$-band light curve of the Mira-type variable in IC 1613 . The period is 640.7 days. The symbols are identical to those in Fig. 1.

derived by Feast et al. (1989) for LMC Miras. At long periods $(P>400$ days $)$, many LMC Miras show such behavior and the relation appears to break down. It is possible that the situation in IC 1613 is similar. 


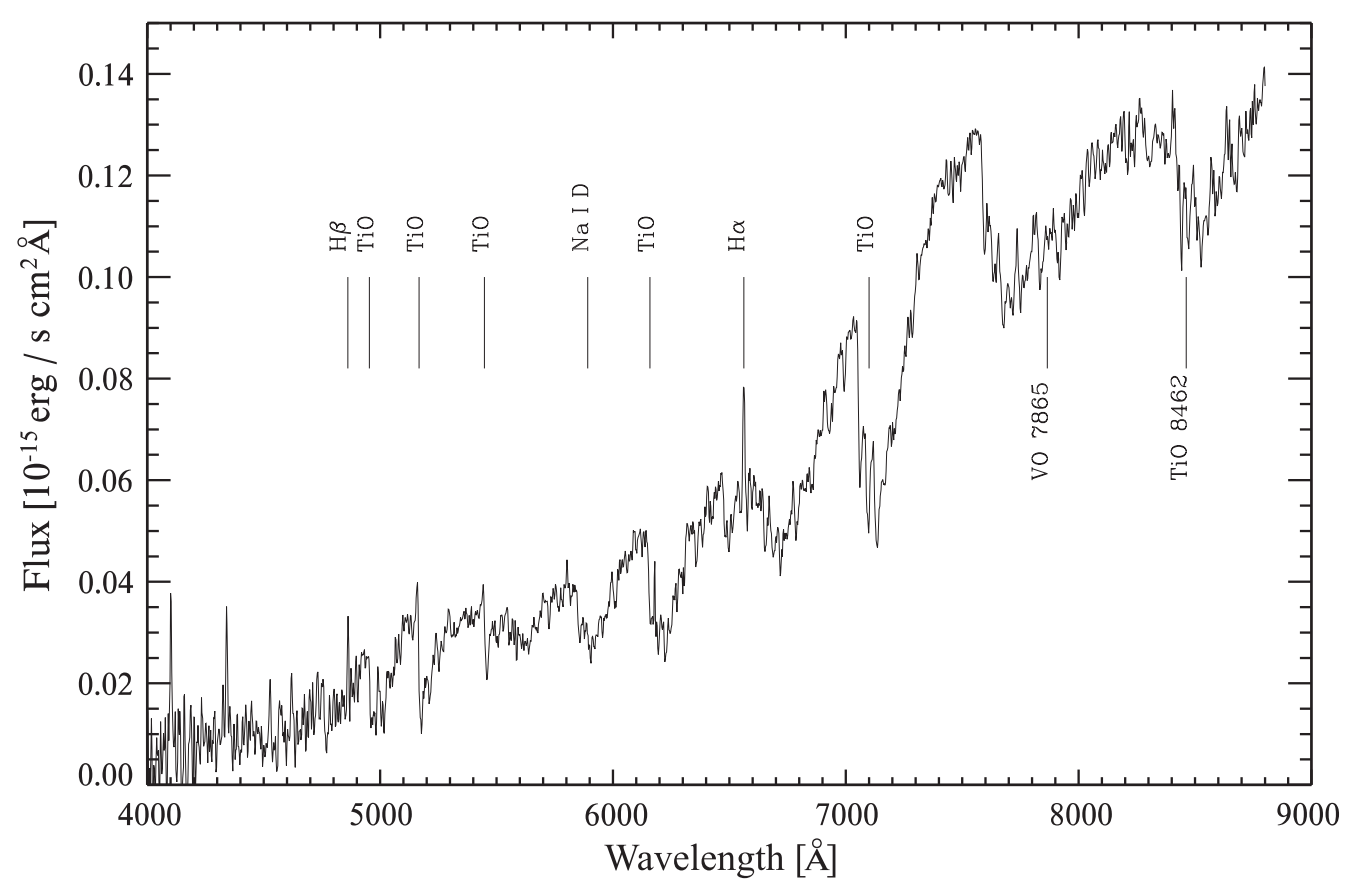

Fig. 3. Keck-II optical spectrum of the Mira variable star.

\section{Spectral classification}

The spectra of very red stars are dominated by bands of TiO and other molecules, including VO. Figure 3 shows the spectrum of the 1999 Nova with the main spectral features marked. The depth of the bands is proportional to the temperature (spectral class) of the star. To quantify this relation, O'Connell (1973) introduced spectral indexes defined as

$$
\begin{aligned}
{[\mathrm{TiO}]_{1}=} & -2.5 \log \left(F_{6180} /\left[F_{6125}+\left(F_{6370}-F_{6125}\right)\right.\right. \\
& \times(6180-6125) /(6370-6125)]) \\
{[\mathrm{TiO}]_{2}=} & -2.5 \log \left(F_{7100} /\left[F_{7025}+\left(F_{7400}-F_{7025}\right)\right.\right. \\
& \times(7100-7025) /(7400-7025)]) \\
{[\mathrm{VO}]=} & -2.5 \log \left(F_{7865} /\left[F_{7400}+\left(F_{8050}-F_{7400}\right)\right.\right. \\
& \times(8050-7865) /(8050-7400)]) .
\end{aligned}
$$

The fluxes are measured in $30 \AA$ bandpasses centered at the corresponding wavelengths. The positions of the bandpasses for the two TiO indexes are shown in Figs. 4a and 4b. Based on several standard stars, Kenyon \& Fernandez-Castro (1987) obtained a calibration of these indexes in the $\mathrm{K}$ and $\mathrm{M}$ spectral classes. Similar calibration but for the $\mathrm{TiO}$ band at $8462 \AA$ was used by Zhu et al. (1999). We will call this index $[\mathrm{TiO}]_{3}$.

To determine the spectral type of the star we first corrected the spectrum for the radial velocity of IC 1613, $-238 \mathrm{~km} \mathrm{~s}^{-1}$. Then we measured the flux at the bandpasses for each index. The results are given in Table 2, together with the quantity ST (see Eqs. (5)-(7) of Kenyon \& Fernandez-Castro 1987 and Eq. (1) of Zhu et al. 1999) and the corresponding spectral class.

The relatively early spectral type given by the $[\mathrm{TiO}]_{1}$ index is due to an "emission" feature at $6180 \AA$ which falls
Table 2. Spectral indexes and classification.

\begin{tabular}{lccc}
\hline Index & Value & $S T$ & Sp. Class \\
\hline$[\mathrm{TiO}]_{1}$ & 0.43 & 2.3 & M2 \\
{$[\mathrm{TiO}]_{2}$} & 0.53 & 2.7 & M3 \\
{$[\mathrm{TiO}]_{3}$} & 0.13 & 3.3 & M3 \\
{$[\mathrm{VO}]$} & 0.13 & 3.0 & M3 \\
{$[\mathrm{Ca} \mathrm{II}]$} & 0.20 & & \\
\hline
\end{tabular}

in the bandpass and decreases the index. The other three indexes give a consistent spectral type, M3. The determination of the luminosity class is much more uncertain. All of the proposed indicators are applicable to an M3 star, or the resolution of our spectrum is not sufficiently high to measure them. The only measurable index sensitive to the luminosity is Ca II $\lambda 8542$ (O'Connell 1973). The value of the Ca II index plotted in Fig. 7 of O'Connell (1973) falls in the region of giants and supergiants. It is not possible to distinguish between the M3I and M3III luminosity classes. Borissova et al. (2000) estimated the bolometric magnitude of the star to be -6.65 , based on $J K$ photometry, suggesting luminosity class I. The star also shows hydrogen Balmer lines in emission, so the spectral class should be M3Ie or M3IIIe.

\section{Is the star a Mira?}

The red variables are classified as Miras, semiregular, and slow irregular variables. Our light curve covers only one maximum well, and the classification of the star into one of these groups based on the photometry alone is not obvious. We know that the star has a period of 640.7 days. 

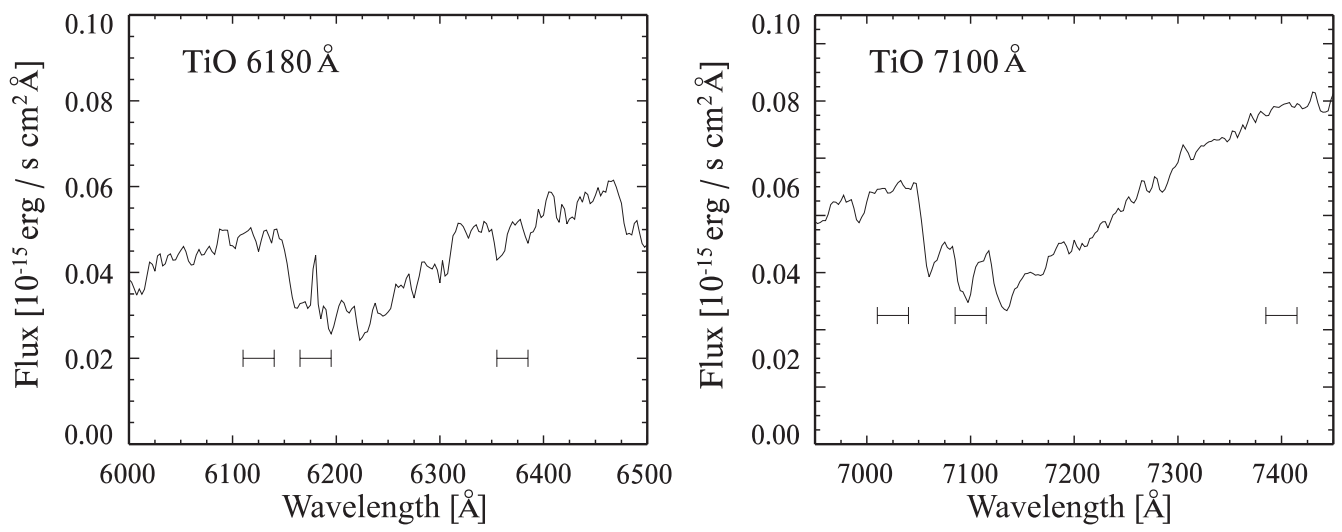

Fig. 4. TiO molecular bands at a) $6180 \AA$ and b) $7100 \AA$. The bars show the intervals used to construct the indexes.

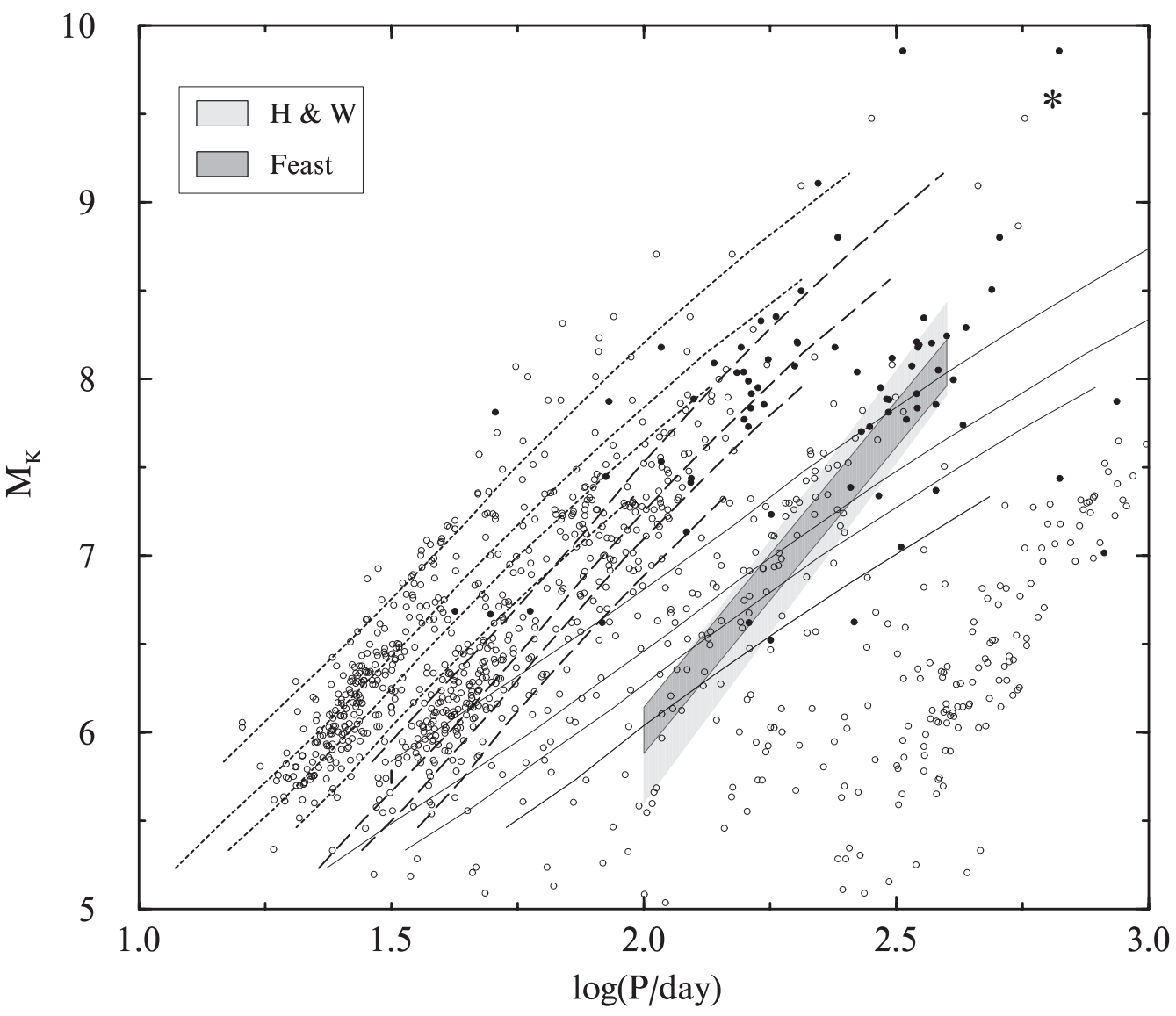

Fig. 5. This is Fig. 6 of Barthes \& Luri (2001). It represents the PL distribution of red variable stars of the LMC in the MACHO data base. The magnitudes in this figure are taken from Wood (1999). The calibrated models are given: fundamental mode (solid lines), first overtone (long-dashed lines), and second overtone (dashed lines). The Mira-like PL relations of Feast et al. (1989) and Hughes \& Wood (1990; H \& W) are also shown. The investigated variable star (asterisk) falls in the zone of the first-overtone pulsating Miras in the LMC.

The $R$ amplitude of the star is around 2.5-3 mag and the light curve is very asymmetric (Fig. 2). Following Mattei et al. (1997), we could reject the semiregular and slow irregular classifications because of the large amplitude of the variations. Plotting the star in Fig. 2 of Mattei et al. (1997), it falls in the region occupied by M-type and S-type Miras. Mennessier et al. (1997) classified the red variables in our Galaxy based on several criteria. We have three of them: the amplitude, the period, and the asymmetry of the light curve. The large amplitude and the long period again put the star between M and S-type Miras. But the light curve is quite asymmetric (the rising part is about $30 \%$ of the period); hence, the star is most probably an M-type Mira.

As mentioned above, the bolometric magnitude of the star is -6.65 . The PL relation for Miras (Hughes \& Wood 1990) is

$\left\langle M_{\text {bol }}\right\rangle=-3.22-7.76[\log (P /$ day $)-2.4] \pm 0.38$, 
from which we predict a bolometric magnitude of $-6.37 \pm$ 0.38. Including also uncertainties in the period and photometric magnitude, the two estimates are completely consistent.

In Fig. 5, we plot the star in Fig. 6 of Barthes \& Luri (2001) representing the PL distribution of LMC red variable stars in the MACHO data base. Observational data are compared to the calibrated models, and the Mira-like PL relations of Feast et al. (1989) and Hughes \& Wood (1990) are also shown. As can be easily seen, the variable star (asterisk in Fig. 5) falls in the zone of the firstovertone pulsating LMC Miras.

We can conclude that "Nova 1999" is a normal longperiod M-type Mira variable star. This is the first confirmed Mira star in IC 1613. The similarity between this star and the LMC Miras suggests that the metallicity does not play an important role in the physics of the pulsation of the Miras (Feast 1996).

Acknowledgements. The authors gratefully acknowledge the useful comments and suggestions made by referees Drs. E. Antonello and L. Mantegazza. The W. M. Keck Observatory is operated as a scientific partnership between Caltech, the University of California, and NASA; it was funded by the generous contributions of the W. M. Keck Foundation. We thank Tom Matheson for calibrating the Keck spectrum. Financial support for this work was provided to A.V.F. by NSF grant AST-9987438 and by the Guggenheim Foundation. KAIT was made possible by donations from Sun Microsystems Inc., the Hewlett-Packard Company, AutoScope Corporation, Lick Observatory, the US National Science Foundation, the University of California, and the Sylvia and Jim Katzman Foundation. This research was supported in part by the Bulgarian National Science Foundation grant under contract No. F-812/1998 with the Bulgarian Ministry of Education and Sciences.

\section{References}

Antonello, E., Fugazza, D., Mantegazza, L., Bossi, M., \& Covino, S. 2000, A\&A, 363, 29

Antonello, E., Mantegazza, L., Fugazza, D., \& Bossi, M. 1999, A\&A, 350, 797

Armandroff, T., \& Massey, P. 1985, ApJ, 291, 685
Baade, W. 1928, Astr. Nach., 234, 407

Barthes, D., \& Luri, X. 2001, A\&A, 365, 519

Borissova, J., Georgiev, L., Kurtev, R., Rosado, M., et al. 2000, RMA\&A, 36, 151

Carlson, G., \& Sandage, A. 1990, ApJ, 352, 587

Cassaly, M., \& Hawarden, T. 1992, JCMT-UKIRT Newsletter, No. 3,33

Dolphin, A., Saha, A., Skillman, E., Tolstoy, E., et al. 2001, ApJ, 550, 554

Feast, M. 1996, MNRAS, 278, 11

Feast, M., Glass, I., Whitelock, P., \& Catchpole, R. 1989, MNRAS, 241, 375

Filippenko, A. V. 1982, PASP, 94, 715

Filippenko, A. V., Li, W. D., Treffers, R. R., \& Modjaz, M. 2001, in IAU Symp. 183, Small Robotic Telescopes, ed. W. Chen (San Francisco: ASP), in press

Freedman, W. 1988a, ApJ, 326, 691

Freedman, W. 1988b, AJ, 96, 1248

Fugazza, D., Mantegazza, L., Poretti, E., \& Antonello, E. 2000, IBVS, 4942

Hughes, S., \& Wood, P. 1990, AJ, 99, 784

Kenyon, S., \& Fernandez-Castro, T. 1987, AJ, 93, 938

King, J. Y., Modjaz, M., \& Li, W. D. 1999, IAUC, No. 7287

Lafler, J., \& Kinman, T. 1965, ApJS, 1, 216

Landolt, A. 1992, AJ, 104, 1

Li, W. D., et al. 2000, in Cosmic Explosions, ed. S. S. Holt, \& W. W. Zhang (New York: AIP), 103

Mantegazza, L., Antonello, E., Fugazza, D., Bossi, M., \& Covino, S. 2001, A\&A, 367, 759

Mattei, J., Foster, G., Hurwitz, L., et al. 1997, Proceedings of the ESA Symposium Hipparcos - Venice '97, 13-16 May, Venice, Italy, ESA SP-402 (July 1997), 269

Mennessier, M., Boughaleb, H., \& Mattei, J. 1997, A\&AS, 124, 143

O'Connell, R. W. 1973, AJ, 78, 1074

Oke, J. B., \& Gunn J. E. 1983, ApJ, 266, 713

Oke, J. B., et al. 1995, PASP, 107, 375

Riess, A., Filippenko, A. V., Li, W., Treffers, R., et al. 1999, AJ, 118, 2675

Sandage, A. 1971, ApJ, 166, 13

Stetson, P. 1993, DAOPHOT II: The Next Generation, MIDAS Users' Manual (ESO, Garching)

Wolf, M. 1906, MNRAS, 67, 91

Wood, P. 1999, Pub. Astron. Soc. Austr., 17 (1), 18

Zhu, Z., Friedjung, M., Zhao, G., Hang, H., \& Huang, C. 1999, A\&AS, 140, 69 